\title{
Design and Development of DRAGONS In-situ Orbital Debris Detection and Characterization Payload*
}

\author{
Haley M. DoYle, Joseph G. Tom, Kari H. NogaceK, Chris R. Anderson, and Jin S. KANG ${ }^{\dagger}$ \\ United States Naval Academy, Annapolis, Maryland, 21402, United States of America
}

\begin{abstract}
Orbital debris poses a constant threat to satellites. Some larger debris can be tracked and avoided, but smaller objects cannot be tracked. In addition, there exists a data gap in orbital debris dispersion models for particles less than $5 \mathrm{~mm}$ in diameter. The Debris Resistive/Acoustic Grid Orbital Navy Sensor (DRAGONS) fills this critical gap in debris monitoring capability. DRAGONS is a sensor system developed by the U.S. Naval Academy and NASA. Consisting of multiple sensor layers, the sensor is capable of providing size, velocity, angle of incidence, and density information of the impacting micrometeoroid and orbital debris. DRAGONS will provide real-time monitoring of orbital debris of small sizes, and will be deployed on the outside of the International Space Station in 2016 for in-situ characterization of debris flux, resulting in improved risk assessment and situation awareness. DRAGONS has been developed and tested for functionality. However, some concerns were raised in regards to its sensitivity to the possible damage that may occur due to environmental loading. The paper provides an overview of the design of the sensor grid, and discusses test results for thermal and vibration loading, as well as potential secondary damage from the force of particle impact.
\end{abstract}

Key Words: NASA, Orbital Debris, MMOD, Sensor, Resistive Grid

\section{Introduction}

Small and untracked orbital debris severely threatens space assets and creates a dangerous environment for manned spacecraft and astronauts. The exact number of debris and their dispersion is difficult to quantify. The U.S. Space Surveillance Network currently tracks orbital debris particles greater than $10 \mathrm{~cm}$ in diameter. However, an estimated tens of millions of particles smaller than $1 \mathrm{~cm}$ in diameter are untracked, presenting a severe impact threat to all assets in space. ${ }^{1)}$ In the early 1980s, NASA developed the Orbital Debris Engineering Model (ORDEM) to map orbital debris. ${ }^{2)}$ While this model is widely used for predicting orbital data environment in space, it lacks data on particles less than $5 \mathrm{~mm}$ in diameter, resulting in inaccuracy. Improving the ORDEM and increasing its accuracy are vital to the safety of many satellites in orbit, present and future.

Orbital debris refers to manmade objects, with the majority composed of explosive fragments, solid rocket motor slag, paint flakes, satellite fragmentation, etc. Micrometeoroid is another form of impact hazard in space, consisting of natural particles such as dust. Particles traveling at hypervelocity (over $5 \mathrm{~km} / \mathrm{s}$ ) can damage solar panels, disrupt electronics, or cripple entire satellite systems. Anything greater than $1 \mathrm{~cm}$ in diameter has the potential to cause catastrophic damage to the International Space Station (ISS). The potential for damage to spacecraft, subsystems, or astronauts is un-

(C) 2016 The Japan Society for Aeronautical and Space Sciences

*Presented at the 30th International Symposium on Space Technology and Science, 4-10 July 2015, Kobe, Japan

Received 19 May 2015; final revision received 19 December 2015; accepted for publication 23 March 2016

†Corresponding author, kang@usna.edu predictable and must be quantified in order to protect human lives and valuable resources.

Over the past few years, work has been done in order to increase the accuracy of ORDEM in order to improve risk assessment and collision avoidance capability. In the earlier stage, in-orbit micrometeoroid and orbital debris (MMOD) sample methods were employed. National Space Development Agency of Japan (NASDA)'s ISS experiments called Micro-particle Capturer (MPAC) and Space Environment Exposure Device (SEED) are examples of such on-orbit data collection efforts. ${ }^{3)}$ MPAC was made of aerogel and polymide foam for particle capture, and had an aluminum backplate for crater counting. Three units were installed on the ISS in 2001 and each unit was retrieved at approximately one-year intervals, in 2002, 2003, and 2004. Although the experiment provided valuable data, it lacked in-situ data gathering capability where the sensors had to be returned to Earth before the data can be obtained.

In order to obtain in-situ MMOD environment characterization, sensors utilizing a resistive grid have been introduced. This method utilizes thinly spaced conductive lines on a flat surface. When an MMOD impact occurs, it creates holes in the lines, causing a change in the overall resistance of the sensor grid. This type of sensor for in-situ MMOD detection is currently under development by both NASA and JAXA. One of the earlier iterations of the sensor by JAXA is currently onboard Horyu-II and QSAT-EOS small satellites. $^{4)}$

NASA and the U.S. Naval Academy (USNA) proposed solution to filling the data gap in the ORDEM is the Debris Resistive/Acoustic Grid Orbital Navy Sensor (DRAGONS). It uses a similar resistive grid sensor for MMOD detection, but it also improves upon previous designs by adding addi- 
tional detection layers, as well as acoustic sensors (to detect lateral acoustic strain waves) for increased sensing capability. The DRAGONS objective is to demonstrate an inexpensive, low-resource space instrument for real-time monitoring of orbital debris, as well as perform data collection for model development. Monitoring the flux, size, velocity, particle density, and location of debris on orbit is required for accurate risk assessment and improved situational awareness by improving ORDEM. ${ }^{5)}$ Real-time information of orbital debris will provide situational awareness in space, alerting the ISS and other space assets to hazards, and potentially saving billions of dollars, as well as human lives in orbit. The current iteration is a proof of concept where the detection range will be limited only to that of the ISS orbit. The current plan by NASA and USNA is to deploy these sensors on CubeSat satellite platforms into various orbits in order to increase the overall accuracy of ORDEM.

When deployed on the ISS in 2016, DRAGONS will be capable of providing real-time, in-situ characterizations of the small-particle orbital debris environment. It will have the ability to detect particles significantly smaller than ground radars are capable of tracking. Design and functionality tests have been performed to validate the sensor performance, including hypervelocity tests. ${ }^{6)}$ However, there were concerns regarding the sensor's sensitivity to thermal fluctuations, launch vibration environment, and a secondary, induced damage due to particle impact. In order to characterize these potential problems, a series of tests have been performed to validate the design.

This paper provides an overview of the sensor grid design, and discusses tests performed and their results in characterizing the sensor grid sensitivity to the potential problems mentioned above. Test data and iterations on the design are described in detail. In addition, the design, development, and testing of the DRAGONS payload for the ISS are described.

\section{Operational Concept}

The DRAGONS sensor will be a passive, three-layer unit covering an area of approximately $1 \mathrm{~m}^{2}$. It will be mounted to the Flight Releasable Attachment Mechanism (FRAM), located on the ISS's Express Logistics Carrier No. 2, facing in the ram-direction, as shown in Fig. 1. The layers will be congruently positioned to receive particle impact. Each layer produces a set of fundamental data that, when assessed, yields information about the size, speed, and density of the impacting body. The depiction of this multiple-layer concept is shown in Fig. 2. The first layer employs a network of resistors, and uses acoustic sensors mounted equidistant in the four corners in conjunction with the second layer, while the third layer serves as a backstop to protect the FRAM.

Over the course of its operational lifetime, DRAGONS will collect data through particle impact, and will relay information about debris to the NASA Orbital Debris Program Office. NASA will use the data to enhance the ORDEM and to make informed recommendations to space-faring entities regarding debris and collision avoidance. Updates

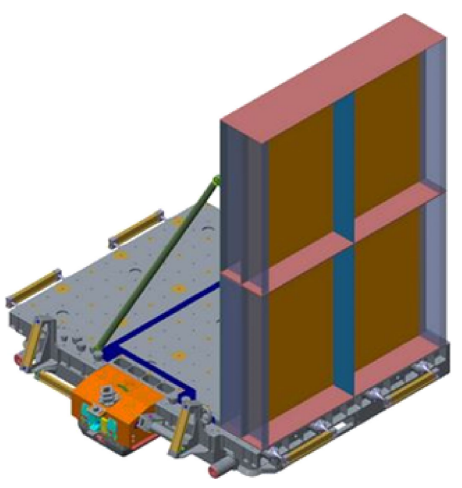

Fig. 1. Depiction of DRAGONS module mounted vertically to FRAM.

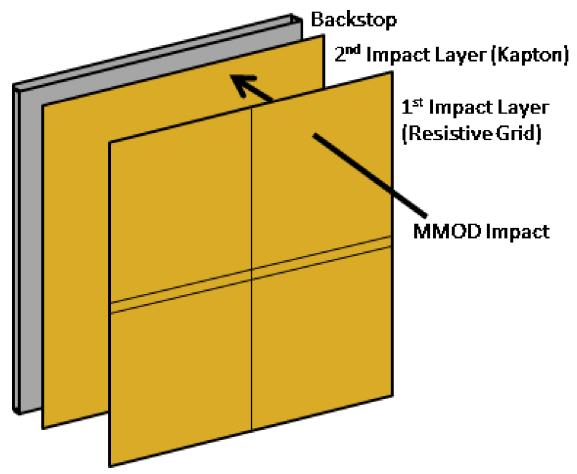

Fig. 2. Depiction of the three-layer design concept.

to the ORDEM will be helpful in directing the ISS and other space assets away from more particle-dense areas of lowearth orbit (LEO), and will bolster their safety and situational awareness. The three-layer sensor test article has been built and tested. Final flight model construction is currently under way, and will be built and launched to the ISS in 2016.

\section{Grid Sensor Design Overview}

\subsection{Three-layer sensor design}

The first layer is a nickel-chromium aluminum silicon (NCAS) grid with a series of eight resistive sub-grids, as shown in Fig. 3. The grid is split into four quadrants, each containing two sub-grids. The sub-grids are labeled A1, A2, B1, B2, C1, C2, D1 and D2, as shown in Fig. 4. Each sub-grid is composed of 818 parallel lines of resistors, measuring about 400 ohms of net resistance per grid, which are etched onto the top surface of a $25 \mu$ m-thick sheet of Kapton. Each line is $250 \mathrm{~mm}$ long, $75 \mu \mathrm{m}$ wide, and spaced $150 \mu \mathrm{m}$ on center.

The overall resistance of the sub-grid increases as debris severs lines of resistors. The measured resistance change is used to determine the number of broken lines, which yields the size of the impacting body. The sub-grids are connected via a copper bus embedded on the grid, and their terminal ends are copper tabs protruding from its edge. The resistance of each sub-grid is measured via wires soldered to each subgrid's corresponding tab and bus, allowing eight resistive measurements to be taken at one time. Attached to the under- 


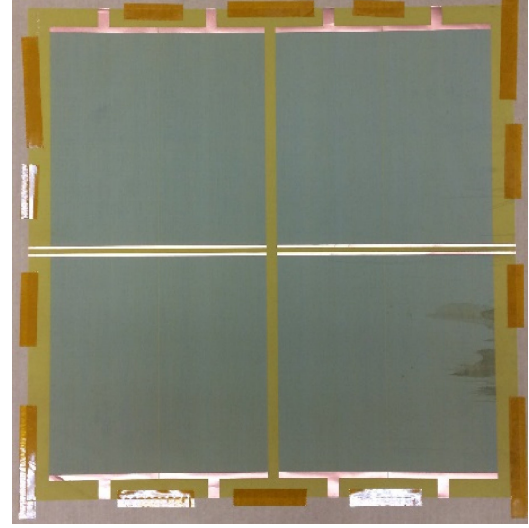

Fig. 3. NCAS grid used for the first layer of DRAGONS unit.

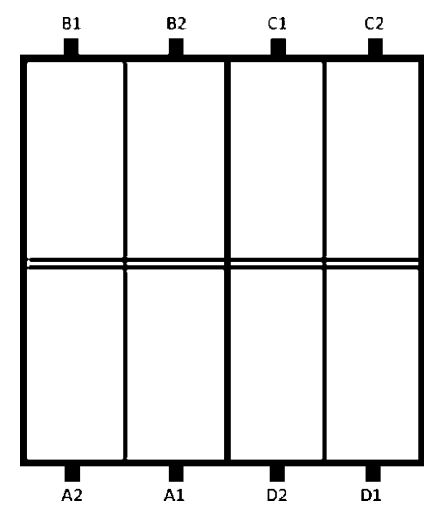

Fig. 4. NCAS grid as divided into sub-grids

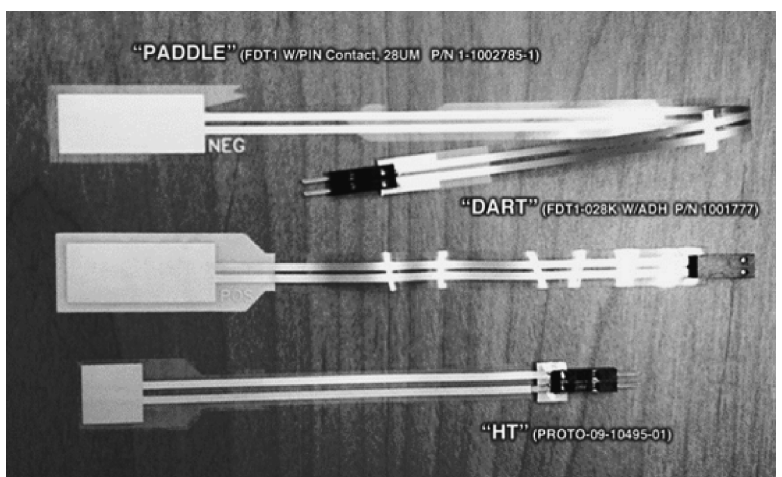

Fig. 5. Picture of acoustic sensors.

side of the Kapton sheet are four polyvinylidene difluoride (PVDF) acoustic strain sensors. Sensors are placed 2 in from each corner of the Kapton sheet. Figure 5 shows a picture of the acoustic sensors. Among the three, the "HT" type was used for DRAGONS because it is designed to resist delamination at high temperatures. An acoustic sensor, as mounted on the backside of the resistive grid, is shown in Fig. 6. An impact through the sheet creates an acoustic wave that the sensors detect using piezoelectric strain transducers. The specific time and location of each impact can be triangulated based on the acoustic signal, detecting a $50 \mu \mathrm{m}$ particle within $3 \mathrm{~cm}$ of the impact location. This concept is depicted in Fig. 7. Redundancy is needed to confirm that changes in resistance are due to impact, rather than unexpected or inci-

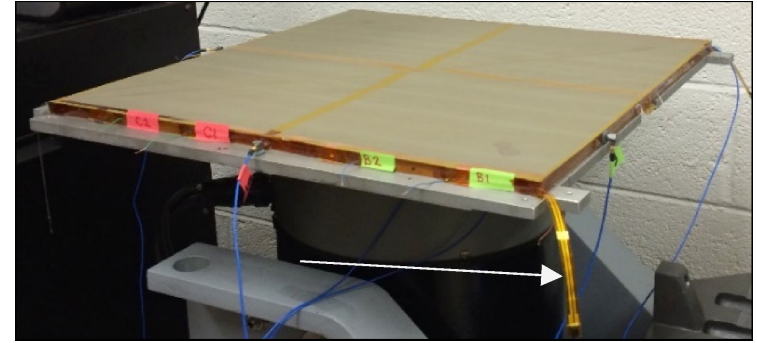

Fig. 6. Acoustic sensors (shown by arrow) mounted to the back side of a sensor grid.

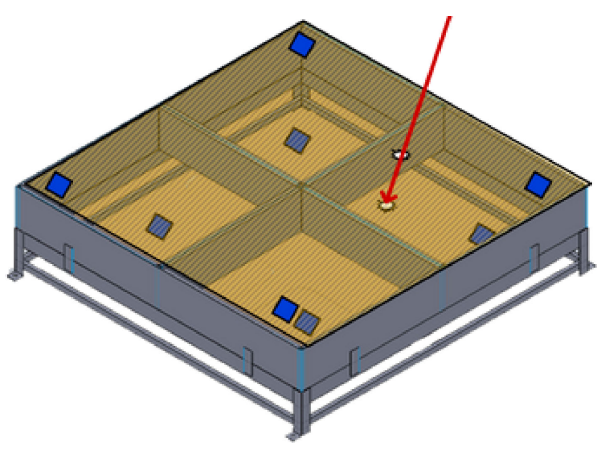

Fig. 7. Depiction of particle impact on the grid sensor.

The arrow denotes particle impact, and the four squares (per layer) near the corners show the placement of the acoustic sensors.

dental changes in resistance. The acoustic sensor detection serves as a redundancy measure to confirm impact on the Kapton sheet.

The second layer sits $15 \mathrm{~cm}$ behind the first layer, and consists of a $25 \mu \mathrm{m}$-thick Kapton sheet with four PVDF sensors in each corner, similar to those placed on the underside of the resistive grid. The sensors will serve the same purpose: to detect acoustic waves created by particle impacts. When the second impact from the same particle occurs as the particle travels through the layers, these sensors record a second time and location. The time interval between the first and second impacts, along with the change in impact location, will provide the velocity and angle of incidence of the particle, as well as confirm the hit for redundancy purposes.

The third layer is a $12.7-\mathrm{mm}$ thick polycarbonate backstop plate. As shown in Fig. 2, it will be placed $25.4 \mathrm{~mm}$ below the second layer, and protect the electronics housing, wires, and the FRAM from particle impacts. It should be noted that the first two layers have an abundance of wiring emanating from tabs, busses, and acoustic sensors. These wires lead to an electronics housing unit that is susceptible to the space environment. The polycarbonate plate will shield these components from particles travelling at high velocities, and will protect the FRAM from potentially devastating impact.

\section{Testing Sensor for Sensitivity to Damage}

The NCAS resistive material is incredibly sensitive and fragile. If anything scrapes, jolts, or even touches the surface of the resistive grid, lines of resistors may break, changing the resistance of the grid. During the handling of the grid, 


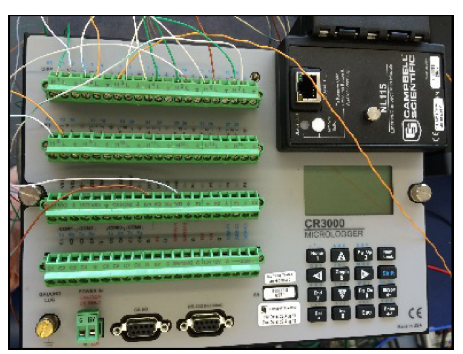

Fig. 8. CR3000 data logger used to track resistive changes.

a large change in grid resistance measurement has been noted. A detailed damage assessment has been conducted where a protective structural "cap" has been designed to minimize physical contact with the grid sensor.

A major concern was raised by the development team regarding sensitivity of the grid to non-impact damage and other environments. Three main areas of concern were identified and tests have been conducted to characterize the sensor sensitivity. These areas were thermal loading, launch vibration loads, and mirror impact data where shock waves created from an impact in one of the sub-grids would cause responses (line breaks) in other sub-grids.

\subsection{Thermal testing for grid material selection}

Two resistive grid candidates and their supporting structures were subjected to thermal testing to verify that the grids will survive and function through the most extreme temperatures they will encounter in orbit. Two materials, nickel chromium $(\mathrm{NiCr})$ and $\mathrm{NCAS}$, were considered for the DRAGONS grid. This testing was done through thermal cycling, with temperatures oscillating between hot and cold "soaks" to simulate the unit being in direct sunlight and Earth's shadow. Testing was completed using the thermal chamber at the USNA's Satellite Instrumentation and Testing Lab. The first thermal tests were done using a NiCr resistive grid taped to a generic aluminum frame. Each tab and bus was connected to a CR3000 data logger through soldered wires, allowing it to take resistive measurements of each of the eight sub-grids continuously throughout the test at $5 \mathrm{~Hz}$, shown in Fig. 8.

Five thermocouples were attached to the "backside" of the sensor grid/frame assembly, as shown in Fig. 16. Multiple thermocouples spanning the sensor surface were used in order to ensure consistent temperatures across the sensor during testing. The grid was cycled through three hot and three cold soaks of one hour each, with the hottest temperature at $111^{\circ} \mathrm{C}$ and the coldest being $-74.5^{\circ} \mathrm{C}$, determined from the expected temperature extremes on the surface of the ISS, as outlined in the ISS interface manual. ${ }^{7)}$

The test results showed that the $\mathrm{NiCr}$ material resistive grid readings fluctuated directly with temperature. As temperatures increased, the resistances rose across the grid. As temperatures fell, resistances fell. While this was an expected behavior, the range of fluctuation was too large for the desired operation conditions, as shown in Fig. 9, with sub-grids resistance values changing between 20 to $30 \mathrm{ohms}$ at the extremes. This corresponds to an equivalence of 50 lines of

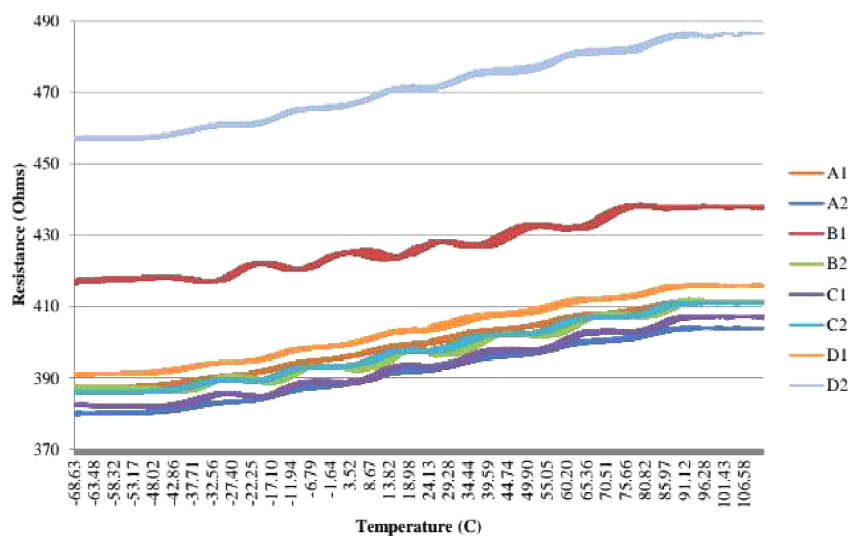

Fig. 9. Resistance of NiCr grid versus temperature. It shows data of hysteresis from three hot/cold soaks.

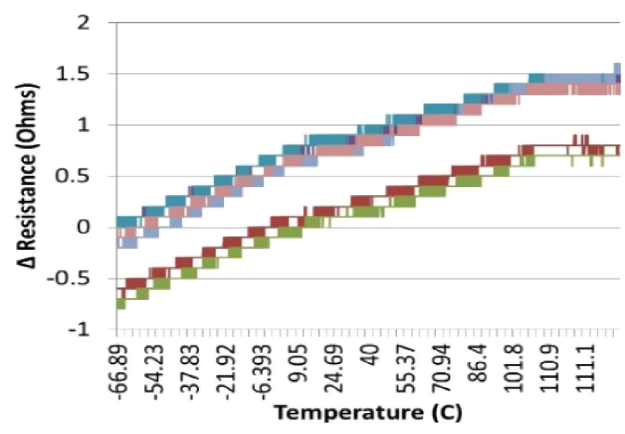

Fig. 10. Resistance of NiCr grid versus temperature.

Each line represents a different location for a thermocouple. It shows data of hysteresis from three hot/cold soaks.

breakage, which correlates to a large particle impact on the order of $\sim 10 \mathrm{~mm}$. This range of output fluctuation was not acceptable since the requirement for the mission is to be able to detect each line breakage. The data also hinted at possible permanent damage to the $\mathrm{NiCr}$ grids, ultimately resulting in rejection of this material for grid sensor development.

In addition, large discrepancies among each grid sensor tested can also be noted from Fig. 9. This is due to the manufacturer's limitations. The demand on the thinness of the conductive lines was at the limit of current manufacturability, and some small variations could not be avoided. However, the baseline resistance values are normalized, and the measurement technique is independent of the absolute value of the grid resistance. The measurement solely relies on the change in grid resistance.

A second thermal test was set up and run identically to the first, with an NCAS material resistive grid replacing the $\mathrm{NiCr}$ grid. The NCAS resistive grid readings through thermal cycling were much more stable than the $\mathrm{NiCr}$ grid. While the $\mathrm{NiCr}$ grid resistance oscillated and steadily increased, as can be seen in Fig. 9, the NCAS material resistive grid remained stable. The NCAS grid's greatest difference in resistance between hot and cold soaks was $2.8 \mathrm{ohms}$, with an average fluctuation in resistance of $1.7 \mathrm{ohms}$ (Fig. 10). This corresponds to an equivalent change of 3 line breaks within the full temperature range. In addition, NCAS resistance recovered fully when the temperature returns to the initial val- 
ue, exhibiting a stable increase/decrease in resistance according to temperature fluctuation without a permanent change. The NCAS grid demonstrated $10 \%$ of the fluctuation that the NiCr showed through thermal cycling. With much improved stability verified, the NCAS material was chosen for the first layer of DRAGONS.

\subsection{Testing for mirrored impact damage reading}

Being able to measure and understand how the resistive grids respond to impact of different sizes and densities is of vital importance to the DRAGONS sensor. Because orbital debris impact is expected to occur at hypervelocity, accurately relating the size of impacting particles to the number of line breaks, and therefore changes in the resistance of the grid, remains a high priority. Realistic hypervelocity impact tests have been successfully carried out. ${ }^{6}$ The results showed that the sensor was able to accurately assess the size and location of the impact.

The concern is raised for mirrored impact damage reading where an impact elsewhere on the grid will cause secondary damage to other parts of the grid, resulting in false readings. Of vital importance is the study of how each of the sub-grids' quality is affected when impacted. As an example, while only one sub-grid may receive a hit, the fragility of the grid and the waves that propagate across the thin Kapton layer may still have an effect on the other seven untouched sub-grids. In order to test for this, low-velocity impact testing was conducted to confirm the robustness of the resistive grid's response to impact. Low-velocity tests were preferred because the impact causes more damage to the grid upon impact, along with stronger shock waves.

A full-size $(0.5 \mathrm{~m} \times 0.5 \mathrm{~m})$ NCAS material resistive grid was tested. Wires were soldered to each of the eight copper tabs and four buses of the grid, and connected to a CR3000 data logger in order to enable constant four-wire measurements of the eight resistive grid sections throughout the test at a rate of $1 \mathrm{~Hz}$. The resistive grid was positioned orthogonally to an air rifle capable of generating a muzzle velocity of approximately $400 \mathrm{~m} / \mathrm{s}$. Aluminum pellets $0.5 \mathrm{~mm}$ in diameter were fired at the resistive grid. Twelve shots were fired with four sub-grids receiving three shots each. Each shot impacted the sub-grid it was intended for without residual impact on other sub-grids. Figure 11 shows the testing setup, while Fig. 12 outlines the locations of the shots on the grid. The grid was repositioned in between each shot while resistive measurements continued. During the experiment, some damage occurred to one of the sub-grids during the repositioning and alignment due to mishandling of the test article during repositioning. This was noted, but was not determined to invalidate testing results due to the clear, quantifiable damage that can be negated from the resistance measurements.

Results from the low-velocity impact testing validated the intended functionality of the grid. Each shot resulted in a clear jump in resistance for the corresponding sub-grid, while all other sub-grids remained relatively stable. Pellets $0.5 \mathrm{~mm}$ in diameter were expected to break three to four lines of resistors, which would cause a resistance change of about 2 ohms.

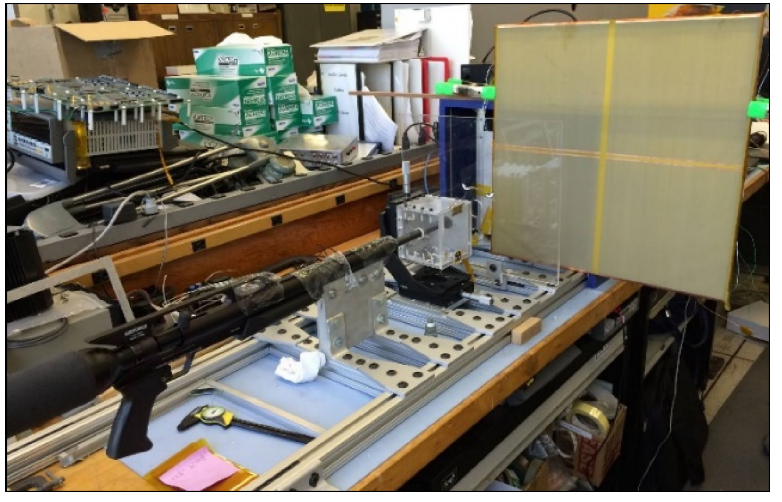

Fig. 11. Low-velocity impact testing setup.

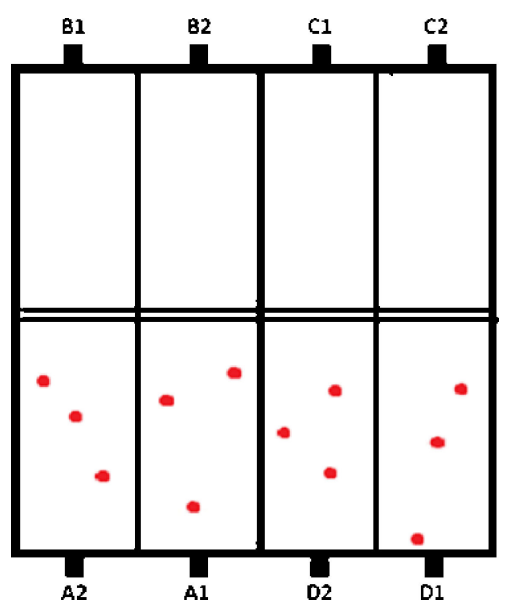

Fig. 12. Locations of low-velocity impact.

Each AL particle that was shot was housed in a Delrin sabot so that the air gun can properly propel the particle round. An intermediate stop-gate is placed to strip the round of the polymer sabot such that only the metal particle continues its trajectory and impacts the sensor grid. However, the setup was not able to completely block all Delrin particles that result from the sabot hitting the stop-gate and being pulverized. This resulted in multiple secondary impacts and dents in the resistive grid, resulting in additional line breakage. An example of such impact is shown in Fig. 13. The effects of secondary impact were also included in the analysis, which still showed consistent data output. With larger pellets than those used in hypervelocity testing, the average resistance change from each impact was $16.4 \mathrm{ohms}$ with a standard deviation of 4.1 ohms. Testing results are displayed in Table 1 below. Each resistance measurement in Table 1 is the result of an average of 30 measurements before and after each shot.

Figure 14 shows a representative plot of the change in resistance resulting from each shot at two particular sub-grids (A1 and A2). Overall, the grids remained relatively stable when untouched, where the fluctuations observed were less than 0.59 ohms. This is a much lower level than actual impact as can be seen in the plot, and is considered negligible. Also of note, many of the minor fluctuations on the sub-grids that were not being shot at throughout the test tended to follow a pattern. This indicates that these small changes in re- 


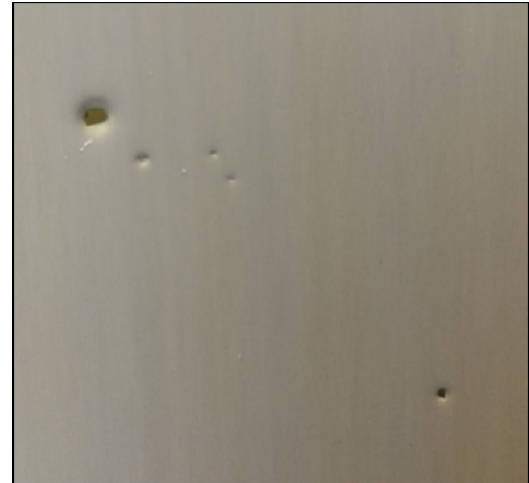

Fig. 13. Example of low-velocity impact on NCAS sub-grid with slight tearing accompanied by smaller dents and impacts due to scattering.

Table 1. Low-velocity resistance change results. All units are in ohms.

\begin{tabular}{cccccc}
\hline Sub-grid & Shot & $\begin{array}{c}\text { Pre-shot } \\
\text { resistance }\end{array}$ & $\begin{array}{c}\text { Post-shot } \\
\text { resistance }\end{array}$ & $\Delta$ & $\begin{array}{c}\text { Standard dev. } \\
\text { (pre-post avg.) }\end{array}$ \\
\hline A2 & 1 & 558.6 & 568.6 & 10.1 & 0.05 \\
& 2 & 568.6 & 584.3 & 15.7 & 1.18 \\
& 3 & 584.7 & 609.1 & 24.4 & 0.02 \\
\hline A1 & 4 & 553.4 & 567.5 & 14.1 & 0.05 \\
& 5 & 567.4 & 582.5 & 15.1 & 0.02 \\
& 6 & 582.4 & 604.1 & 21.7 & 0.03 \\
\hline D2 & 7 & 599.2 & 620.6 & 21.4 & 0.03 \\
& 8 & 584.9 & 599.2 & 14.3 & 0.03 \\
& 9 & 620.6 & 635.3 & 14.8 & 0.05 \\
\hline D1 & 10 & 598.4 & 610.5 & 12.1 & 0.01 \\
& 11 & 610.6 & 629.1 & 18.5 & 0.02 \\
& 12 & 628.9 & 643.6 & 14.6 & 0.74 \\
\hline & & & Ave. $\Delta$ & 16.4 & 0.19 \\
& & & Std. dev. & 4.1 & \\
\hline
\end{tabular}

sistance did not result from resistive line breakage, as it is extremely unlikely that the same number of lines would be broken at the same time in multiple different sections of the grid. These small resistance fluctuations were in both increase and decrease directions. Since resistive lines are incapable of repairing themselves once broken, it can be concluded that these minor fluctuations were not the result of permanent damage to the resistors.

While impact causing resistance changes and small halfohm fluctuations are clearly discernible in low-velocity testing, orbit presents a far more complicated environment than our laboratory testing facilities. A $50 \mu \mathrm{m}$-sized particle could potentially strike the resistive grid in orbit, causing a single line break and a half-ohm change in resistance. In these cases, acoustic sensors will alert the DRAGONS of an impact, complementing the resistive changes in the grid.

Low-velocity impact testing validated that the DRAGONS can detect specific impacts in different sub-grids without damaging other sub-grids, as well as the time and size of the impact. While minor fluctuations occur in each of the grids, they can be distinguished from real particle impact.

In addition to the initial low-velocity testing performed for functionality verification, as described above, actual hypervelocity impact tests were also performed in order to validate

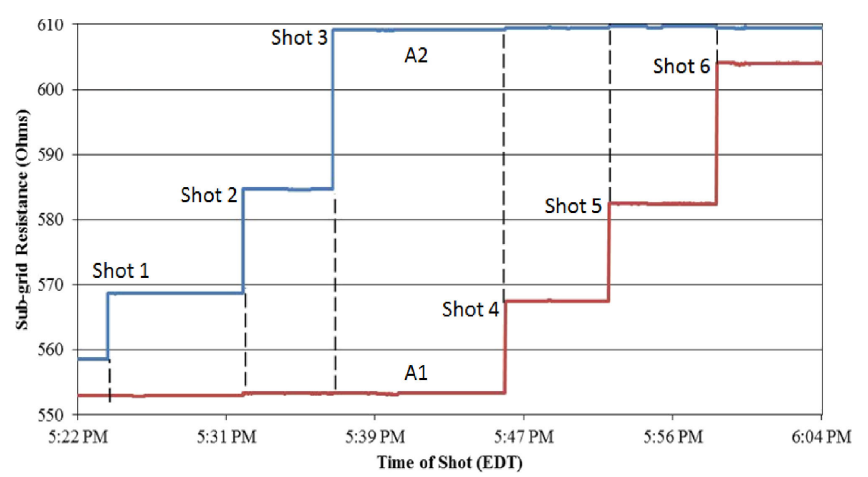

Fig. 14. Sub-grid A1 and A2 reactions to low-velocity particle shots.

the design to the conditions that more closely simulate the impacts that are expected in orbit. The sensor grid was taken to the hypervelocity test facility at the University of Kent in Canterbury, England for impact testing. ${ }^{6}$ ) The hypervelocity testing results confirmed that the sensor grid provides accurate reading of the impact, as well as demonstrates stability in generated data.

\subsection{Launch vibration load testing}

The primary structural design challenge was to secure the thin and sensitive NCAS grid and Kapton sheet layers, holding them stable during system launch and deployment. The holding mechanism cannot damage the layers or interfere with their data collection in any way, is required to hold the sheets taught and allow access for wiring, and attach to the grid and acoustic sensors. The solution was a sheet clamping device that stabilizes the first two layers while not damaging them or hindering their operation.

\section{Clamping system}

Two clamping systems secure the NCAS grid and Kapton sheet (layers one and two, respectively). It is imperative that the failure mode is not the slippage of the layers where the layers may slip out of the holding mechanism. The holding mechanism is able to fasten the grids secure enough so that the layers do not tear before being pulled out.

Each clamp consists of two congruent $6.35 \mathrm{~mm}$-thick aluminum 6061-T6 frames $(58.5 \times 58.5 \mathrm{~cm}$ outer dimensions and $54.6 \times 54.6 \mathrm{~cm}$ inner dimensions). The main element of the clamp is a Viton o-ring that is circumferentially slotted along the underside of the top frame, and compressed against the $25 \mu \mathrm{m}$ layer as it rests on the bottom frame. The slot for the o-ring is $108 \mathrm{~mm}^{2}$, allowing for a ten percent compression of the Viton as the two frames are bolted together. Cross-sections and depictions of the top and bottom components of the clamp are shown in Fig. 15.

In order to validate the clamping concept, a Kapton sheet was suspended between the two cross-sectioned clamping mechanisms and subjected to tension in an MTS machine. After securing the sheet to the $0.5 \mathrm{~mm}$ step on the clamp top frame with Kapton tape and securing the two clamping pieces together, the Kapton withstood $267 \mathrm{~N}$ before tearing at the center. This failure mode, which is a preferable outcome to sheet slippage or clamp deformation, validated that the design will not be the point of failure in holding the grid 

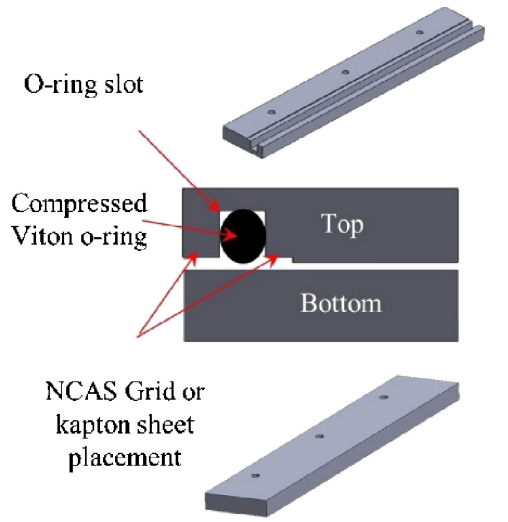

Fig. 15. Cross-sections of clamping system top and bottom frames.

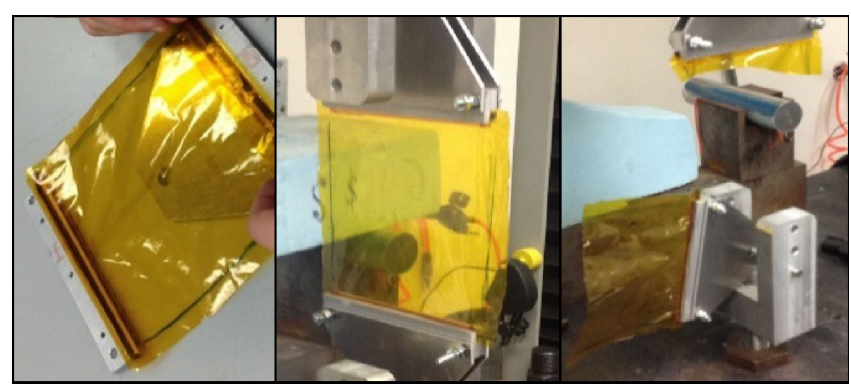

Fig. 16. Kapton sheet is taped into place on the DRAGONS clamp, subjected to tension on the MTS machine.

(Left) Clamp assembly. (Center) MTS machine testing. (Right) Kapton failure.

in place. Figure 16 shows the breakage, and Fig. 17 shows the NCAS grid successfully secured in the DRAGON clamping system.

\section{Test article structure}

Impact and vibration testing for DRAGONS required that each layer's clamp be held in deployment orientation: the second layer $15 \mathrm{~cm}$ directly below the first. A structure was developed to hold the first two layers and polycarbonate backstop in place. Figure 18 shows a CAD rendering of the structure. While the structure would not be mountable on the ISS's FRAM in this configuration, it serves as a development tool for further vibration and impact testing. One small L-shaped aluminum "foot" was bolted to the bottom of each corner to allow the device to be outfitted to the NASA hypervelocity impact testing apparatus at both University of Kent facilities and those at White Sands, New Mexico, where all three layers of DRAGONS have undergone impact testing as a complete unit.

Structure and grid vibration testing

The vibrational survivability of the resistive grid was of primary concern. The grid must survive launch to orbit and show little to no resistive change. A vibrational test of the grid was conducted and the resistance change was measured. A resistive grid was secured to a rectangular aluminum stand, which was bolted down to a slip table via an aluminum adapter plate. Three accelerometers were adhered to each layer to ensure the grid received the correct vibration profile. GSFC-STD-7000A random vibration levels were used for

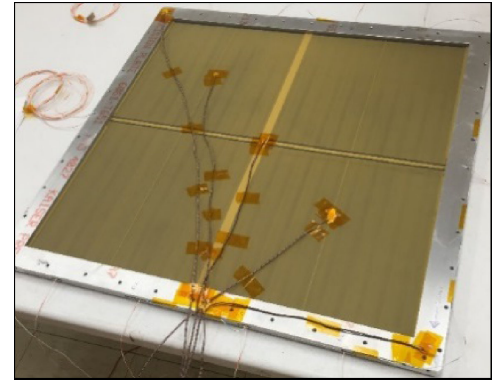

Fig. 17. NCAS grid secured in DRAGONS clamp with thermocouples attached to grid backing.

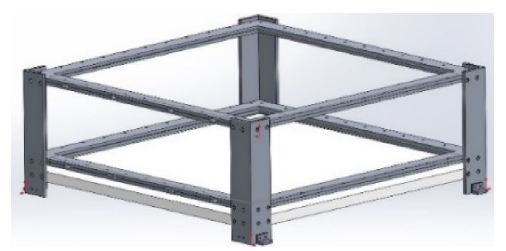

Fig. 18. SolidWorks rendering of DRAGONS test article structure, holding two clamps and polycarbonate backstop.

the test. ${ }^{8)}$

The grid underwent vibration in all three axes, as well as a sine sweep from $20-2,000 \mathrm{~Hz}$ before and after each test. Because the most critical part of the test was to understand the effect on the grids, resistive measurements were taken in between each step of the process, including in between reorienting the grid and before and after each sine sweep and random vibration test. In most cases, the resistance of each sub-grid increased less than $0.5 \mathrm{ohms}$ (the equivalent of one resistive line break) between each random vibration, an acceptable level that demonstrates grid survivability for launch to the ISS.

The major lesson learned from this test was how imperative it will be to protect the face of the resistive grid from any wires, rocket foreign object debris (FOD), or anything else that may endanger it on its way to being mounted on the FRAM. Lengthy protective measures and transportation protection devices will be developed to further ensure grid safety.

\section{Conclusions}

In order to remedy the gap in the orbital debris data for small particles of $10 \mathrm{~mm}$-diameter or less, DRAGONS has been developed. It is a three-layer design that will characterize an orbital debris or micrometeoroid impact by providing the size of the particle, its velocity, direction of travel, and possibly composition. The design was tested for output fluctuation in an extreme thermal environment and survivability under launch vibration loads. A structure was also developed for safe deployment of the sensors in orbit without damaging the sensor grids.

The main functionality of the sensor grid has been successfully tested at hypervelocity impact speeds. However, there were concerns regarding the sensor's sensitivity to ther- 
mal fluctuation, launch vibration environment, and secondary, induced damage due to particle impact. In order to characterize these potential problems, a series of tests were performed to validate the design.

The test data showed that the final design of the grid can clearly provide impact data with a low noise level, as well as be robust enough to be deployed on the ISS without any loss of capability. It is expected that the DRAGONS module will be deployed to the ISS for two years. The sensor payload is expected to encounter 100 to 200 impacts during its lifespan. $^{2)}$

In the future, the DRAGONS concept will be applied to other spacecraft, creating a network of detectors that will provide an accurate map of the orbital debris environment. The sensor will continue to characterize the orbital debris in a multitude of orbits, including NASA's orbits of interest, which have little-known debris environments. DRAGONS could be deployed and scaled to fit on almost any satellite, providing real-time situational awareness. Immediately detecting impacts in orbit will provide other spacecraft information on where and how fast debris is moving, alerting a satellite to reorient or maneuver itself to avoid impact damage. The more DRAGONS sensors are deployed into orbit, the more accurate the Orbital Debris Engineering Model, which is widely used to protect assets in space, will be. This passive sensor will change the way orbital debris is detected, increasing our knowledge of the environment and make space ventures safer.

\section{Acknowledgments}

The United States Naval Academy student design team would like to extend its gratitude to Dr. Christopher Anderson, Dr. Jin Kang, and Ms. Haley Doyle for their assistance in the development and testing of DRAGONS components and modules. The team would also like to thank the engineers at NASA Johnson Space Center in Houston, Texas, who offered counsel and guidance in the design and testing process.

\section{References}

1) Ramsey, M. D., Ash, D. J., Bunker, E. M., Harden, B. D., Musmanno, T. M., Anderson, C. R., Corsaro, R., Burchell, M. J., Stansberry, E. G., and Liou, J.-C.: Debris Resistive Acoustic Grid Orbital Navy Sensor (DRAGONS), AIAA Infotech@Aerospace Conference, St. Louis, MO, May 2012.

2) Krisko, P. H.: The New NASA Orbital Debris Engineering Model ORDEM 3.0, NASA, 2014.

3) Neish, M. J., Imagawa, K., Inoue, T., Ishizawa, J., Kitazawa, Y., Yamaura, Y., Murakami, A., and Ochi, Y.: Microparticle Capture on the International Space Station Using Aerogel and Polyimide Foam, 9th International Symposium on Materials in a Space Environment, Noordwijk, Netherlands, June 2003.

4) Matsumoto, H., Kitazawa, Y., Okudaira, O., Hanada, T., Sakurai, A., Funakoshi, K., Yasaka, T., Akahoshi, Y., and Hasegawa, S.: Space Debris Sensor for In-situ Observation of Micrometeoroid and Orbital Debris in JAXA, 29th Internaional Suposium on Space Technology and Science, Nagoya, Japan, June 2013.

5) Liou, J.-C., Sadilek, A., Burchell, M., Corsaro, R., and Giovane, F.: DRAGONS-A Micrometioroid and Orbital Debris Impact Sensor, NASA Orbital Debris Program Office, USA, US Naval Academy, USA, University of Kent, UK, Naval Research Lab, USA, Virginia Tech, USA, 2012.

6) Tsao, M. A., Corsaro, R., Ngo, H. T., and Anderson, C. R.: An In-Situ Measurement System for Characterizing Orbital Debris, submitted to IEEE Transactions on Instrumentation and Measurement, January 2016.

7) Qualification and Acceptance Environmental Test Requirements (Revision AA), International Space Station Program, Available from: http: //www.nasa.gov, 2010.

8) General Environmental Verification Standard, Revision A, NASA Goddard Space Flight Center, Available from: http://standards.gsfc.nasa. gov, 2013.

\author{
S. Matunaga \\ Associate Editor
}

